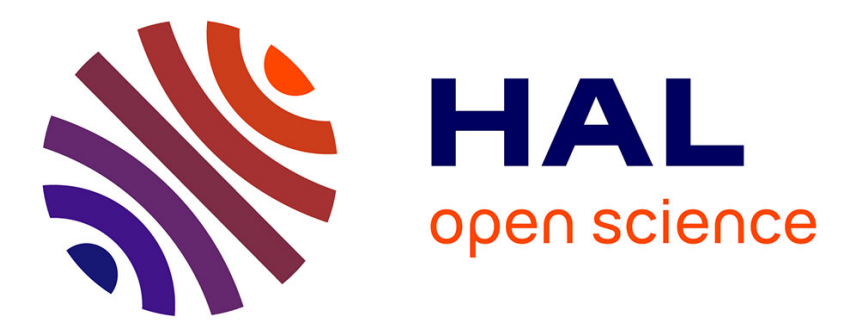

\title{
On a phenomenon of Turán concerning the summands of partitions
}

\author{
Cécile Dartyge, Mihály Szalay
}

\section{To cite this version:}

Cécile Dartyge, Mihály Szalay. On a phenomenon of Turán concerning the summands of partitions. Acta Mathematica Hungarica, 2016, 149 (2), pp.375-395. 10.1007/s10474-016-0623-0 . hal-01280845

\section{HAL Id: hal-01280845 \\ https://hal.science/hal-01280845}

Submitted on 1 Mar 2016

HAL is a multi-disciplinary open access archive for the deposit and dissemination of scientific research documents, whether they are published or not. The documents may come from teaching and research institutions in France or abroad, or from public or private research centers.
L'archive ouverte pluridisciplinaire HAL, est destinée au dépôt et à la diffusion de documents scientifiques de niveau recherche, publiés ou non, émanant des établissements d'enseignement et de recherche français ou étrangers, des laboratoires publics ou privés. 


\title{
On a phenomenon of Turán concerning the summands of partitions
}

\author{
CÉcile Dartyge * Mihály Szalay *
}

February 11, 2016

\begin{abstract}
Turán [12] proved that for almost all pairs of partitions of an integer, the proportion of common parts is very high, that is greater than $\frac{1}{2}-\varepsilon$ with $\varepsilon>0$ arbitrarily small. In this paper we prove that this surprising phenomenon persists when we look only at the summands in a fixed arithmetic progression.
\end{abstract}

\section{Introduction}

For $n \in \mathbb{N}$, let $p(n)$ be the number of partitions of $n$. By Hardy and Ramanujan [9] we have:

$$
p(n)=(1+o(1)) \frac{1}{4 n \sqrt{3}} \exp \left(\frac{2 \pi \sqrt{n}}{\sqrt{6}}\right) .
$$

Turán [12], [13], [14] proved a surprising phenomenon related to the partitions of integers. If we take randomly $k$ partitions of an integer $n$ then in almost all cases with at most $o\left(p(n)^{k}\right)$ exceptions, the proportion of the parts common to all these $k$ partitions is greater than $\frac{1}{k}-\delta$ with $\delta>0$ as small as we want.

For $n \in \mathbb{N}$ and $\Pi(n): n=\lambda_{1}+\cdots+\lambda_{m}, \lambda_{1} \geq \ldots \geq \lambda_{m}$ a partition of $n$, we denote by $\overline{\Pi(n)}=\left\{\lambda_{1}, \ldots, \lambda_{m}\right\}$ the multiset formed by the summands of $\Pi(n)$. Erdős and Lehner [7] proved that for almost all partitions, the number of parts $m(\Pi)$ is $(1+o(1)) \frac{\sqrt{6 n}}{2 \pi} \log n$.

The result of Turán is in fact more general than what we previously recalled. Precisely ([13] Theorem I' p. 192), he proved that for any $\delta>0$, $n(1+o(1)) \leq n_{1} \leq n_{2} \leq \ldots \leq n_{k} \leq n(1+o(1))$, for almost all $k$-tuples $\left(\Pi_{1}\left(n_{1}\right), \ldots, \Pi_{k}\left(n_{k}\right)\right)$ we have:

$$
\left|\overline{\Pi_{1}\left(n_{1}\right)} \cap \ldots \cap \overline{\Pi_{k}\left(n_{k}\right)}\right| \geq\left(\frac{1}{k}-\delta\right) \max \left(\left|\overline{\Pi_{1}\left(n_{1}\right)}\right|, \ldots, \overline{\Pi_{k}\left(n_{k}\right)} \mid\right) .
$$

2010 Mathematics Subject Classification: primary 11P82; secondary 05A17, 11 P83.

* Research partially supported by the Hungarian National Foundation for Scientific Research, Grant K 100291 
The aim of this paper is to examine whether such phenomenon still exists when we consider only the parts in a given arithmetic progression. In the papers [1], [2], [3] it is proved that for almost all partitions, the summands are well distributed in residue classes. In particular in [1] it is proved that if $1 \leq a \leq d$ are fixed integers then for almost all partitions of $n$ the number of summands $\equiv a \bmod d$ is $(1+o(1)) \frac{\sqrt{6 n}}{2 \pi d} \log n$.

In [3] we obtained some results valid for $d \leq n^{1 / 2-\varepsilon}$ but with some limitations due to the fact that the small parts occur frequently. In [4] we in fact proved that there are some "dominant" residue classes, the small classes $1 \bmod d, 2 \bmod d$ appearing more frequently than the class $m$ $\bmod d$ when $m$ is large.

Extending the notations of Turán to this context of arithmetic progressions, we now consider for $1 \leq a \leq d, \overline{(\Pi(n), a, d)}$ the multiset of the summands $\equiv a \bmod d$ from $\Pi(n)$.

Our first result shows that Turán 's phenomenon persists for the parts in a fixed arithmetic progressions.

Theorem 1. Let $k \geq 2$, a, $d$ fixed integers with $1 \leq a \leq d$. Then for $n \rightarrow \infty, n \leq n_{1} \leq n_{2} \leq \ldots \leq n_{k} \leq n(1+o(1)), \delta>0$, for almost all partitions $\Pi_{1}\left(n_{1}\right), \ldots, \Pi_{k}\left(n_{k}\right)$ of $n_{1}, \ldots, n_{k}$ respectively, we have:

$$
\left|\overline{\left(\Pi_{1}\left(n_{1}\right), a, d\right)} \cap \ldots \cap \overline{\left(\Pi_{k}\left(n_{k}\right), a, d\right)}\right| \geq(1-\delta) \frac{\sqrt{6}}{2 \pi k d} \sqrt{n} \log n,
$$

with at most $o\left(p\left(n_{1}\right) \cdots p\left(n_{k}\right)\right)$ exceptions.

Let $\alpha\left(n_{1}, \ldots, n_{k} ; m ; a, d\right)$ denote the number of multisets such that

$$
\left|\overline{\left(\Pi_{1}\left(n_{1}\right), a, d\right)} \cap \ldots \cap \overline{\left(\Pi_{k}\left(n_{k}\right), a, d\right)}\right|=m .
$$

The proof of Theorem 1 is based on an upper bound on

$$
\sum_{m \leq M} \alpha\left(n_{1}, \ldots, n_{k} ; m ; a, d\right)
$$

It would be interesting to have estimates for the individual $\alpha\left(n_{1}, \ldots, n_{k} ; m ; a, d\right)$ at least when $m$ is close to the expected number of common summands. Here we concentrate on the most natural case, that is when $k=2$ and $n_{1}=n_{2}$.

Theorem 2. Let $1 \leq a \leq d$ be fixed. For $\varepsilon>0$ and $M$ satisfying

$$
\left(\frac{3}{4}+\varepsilon\right) \frac{\sqrt{6 n}}{4 \pi d} \log n \leq M \leq \frac{n^{\frac{5}{8}}}{d}
$$

we have:

$$
\begin{aligned}
\alpha(n, n ; M ; a, d) & =(1+o(1)) p^{2}(n)\left(\frac{2 \pi d}{\sqrt{6 n}}\right)^{1-\frac{a}{d}} \frac{1}{\Gamma\left(\frac{a}{d}\right)} \\
& \times \exp \left(-\frac{\sqrt{6 n} \exp \left(\frac{-2 \pi d M}{\sqrt{6 n}}\right)}{2 \pi d}-2 a M \frac{\pi}{\sqrt{6 n}}\right) .
\end{aligned}
$$

A first application is the following estimate on the cardinality of the intersection of two multisets form by two partitions of an integer $n$. 
Corollary 1. Let $1 \leq a \leq d$ be some fixed integers and $\varepsilon>0$. For almost all pairs $\left(\Pi_{1}(n), \Pi_{2}(n)\right)$ of partitions of $n, i$. e. with at most $o\left(p^{2}(n)\right)$ exceptions, we have:

$$
\left(\frac{3}{4}+\varepsilon\right) \frac{\sqrt{6 n}}{4 \pi d} \log n \leq\left|\overline{\left(\Pi_{1}(n), a, d\right)} \cap \overline{\left(\Pi_{2}(n), a, d\right)}\right| \leq \frac{n^{\frac{5}{8}}}{d} .
$$

In [14], Turán posed the following problem ([14] Problem 3 p.319). Let $k \geq 2, \lambda \in \mathbb{R}$ fixed and $n \leq n_{1} \leq n_{2} \leq \ldots \leq n_{k} \leq n(1+o(1))$. Denoting by $K\left(n_{1}, n_{2}, \ldots, n_{k} ; \lambda\right)$ the number of $k$-tuples of unrestricted partitions $\left(\Pi_{1}, \ldots, \Pi_{k}\right)$ of $n_{1}, \ldots, n_{k}$ with property

$$
\left|\overline{\Pi_{1}} \cap \overline{\Pi_{2}} \cap \ldots \cap \overline{\Pi_{k}}\right| \geq \frac{\sqrt{6 n}}{2 \pi k} \log n+\lambda \sqrt{n} .
$$

Is it true that

$$
\lim _{n \rightarrow \infty} \frac{K\left(n_{1}, n_{2}, \ldots, n_{k} ; \lambda\right)}{p\left(n_{1}\right) \cdots p\left(n_{k}\right)}=\Phi(\lambda)
$$

exists?

We can apply Theorem 2 to solve this problem in the case $k=2$ and $n_{1}=n_{2}=n$. Furthermore we can detect the distribution of the number of common parts $\equiv a \bmod d$. Let $K\left(n_{1}, \ldots, n_{k} ; \lambda ; a, d\right)$ be the number of $k$-tuples of unrestricted partitions $\left(\Pi_{1}, \ldots, \Pi_{k}\right)$ of $n_{1}, \ldots, n_{k}$ with the same property as (3) but with each $\overline{\Pi_{j}\left(n_{j}\right)}$ replaced by $\left(\overline{\Pi_{j}\left(n_{j}\right)}, a, d\right)$ and $\frac{\sqrt{6 n}}{2 \pi k} \log n+\lambda \sqrt{n}$ by $\frac{1}{d}\left(\frac{\sqrt{6 n}}{2 \pi k} \log n+\lambda \sqrt{n}\right)$.

Corollary 2. Let $1 \leq a \leq d$ be fixed integer and $\lambda \in \mathbb{R}$ also fixed. We have:

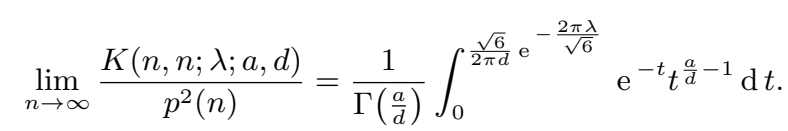

Theorem 2 and its corollaries are valid only for pairs of partitions whereas the general situation of Theorem 1 . We have chosen this particular case in order to facilitate the presentations of the proofs but it doesn't seem to have real difficulty to obtain analogous results for $k$-tuples of partitions.

In [3] and [4] the results are valid for large range for $d$ for example for $d \leq n^{1 / 8-\varepsilon}$. It is probably possible to obtain something interesting for such a region of $d$. Here again we decided to concentrate in case of fixed $d$ in order to avoid some complications in the computations.

\section{On an identity of Turán}

Let $D\left(n_{1}, n_{2}, \ldots, n_{k}, L\right)$ denote the number of $k$-tuples $\left(\Pi_{1}\left(n_{1}\right), \ldots, \Pi_{k}\left(n_{k}\right)\right)$ such that $\left|\overline{\Pi_{1}\left(n_{1}\right)} \cap \overline{\Pi_{2}\left(n_{2}\right)} \cap \cdots \cap \overline{\Pi_{k}\left(n_{k}\right)}\right| \leq L$. Turán [13] Lemma I p. 193 proved for all $0 \leq x_{j}<1$ the following identity:

$$
\sum_{n_{1}=0}^{\infty} \cdots \sum_{n_{k}=0}^{\infty} D\left(n_{1}, n_{2}, \ldots, n_{k}, L\right) \prod_{i=1}^{k} x_{i}^{n_{i}}=\prod_{i=1}^{k} F\left(x_{i}\right) \prod_{\nu=L+1}^{\infty}\left(1-\left(\prod_{i=1}^{k} x_{i}\right)^{\nu}\right)
$$


where

$$
F(r)=\prod_{\nu=1}^{\infty}\left(1-r^{\nu}\right)^{-1}
$$

In this section we adapt this identity of Turán to our context with some arithmetic progression condition. We want to study the generating function associated to the coefficients $\alpha\left(n_{1}, \ldots, n_{k} ; m ; a, d\right)$ :

$$
G(\mathbf{x}, z)=\sum_{n_{1}=0}^{\infty} \cdots \sum_{n_{k}=0}^{\infty} \sum_{m=0}^{\infty} \alpha\left(n_{1}, \ldots, n_{k} ; m ; a, d\right) x_{1}^{n_{1}} \cdots x_{k}^{n_{k}} z^{m},
$$

with the notation $\mathbf{x}=\left(x_{1}, \ldots, x_{k}\right)$.

Adapting Turán 's argument we obtain

Lemma 1. For any $\left|x_{1}\right|<1, \ldots,\left|x_{k}\right|<1,\left|x_{1}^{a} \cdots x_{k}^{a} z\right|<1$ we have:

$$
G(\mathbf{x}, z)=\left\{\prod_{\nu=1}^{\infty} \frac{1}{\left(1-x_{1}^{\nu}\right) \cdots\left(1-x_{k}^{\nu}\right)}\right\}\left\{\prod_{\nu \equiv a} \frac{1-\left(x_{1} \cdots x_{k}\right)^{\nu}}{1-\left(x_{1} \cdots x_{k}\right)^{\nu} z}\right\} .
$$

Proof. We examine the contribution of the parts equal to a given integer $\nu \geq 1$ in the function $G$. Let $\left(\Pi_{1}\left(n_{1}\right), \ldots, \Pi_{k}\left(n_{k}\right)\right)$ be a $k$-tuple of partitions counted in $\alpha\left(n_{1}, \ldots, n_{k} ; m ; a, d\right)$. We may write each $\Pi_{i}\left(n_{i}\right)$ in the way $n_{i}=\sum_{\nu \geq 1} h_{\nu}^{(i)} \nu$ where $h_{\nu}^{(i)}$ denotes the number of parts equal to $\nu$ in the partition $\Pi_{i}\left(n_{i}\right)$.

If $\nu \not \equiv a \bmod d$ then any multiplicity $h_{\nu}^{(i)}$ in partitions of the $n_{i}$ is possible when $n_{i}$ is running over all the positive integers. In other words the terms with part $\nu$ will appear in the form $x_{1}^{h_{1} \nu} \cdots x_{k}^{h_{k} \nu}$ with $h_{1}, \ldots, h_{k} \geq 0$. Thus the contribution of the $\nu \not \equiv a \bmod d$ brings to $G$

$$
g_{1}(\nu):=\prod_{i=1}^{k}\left(\sum_{h_{i}=0}^{\infty} x^{\nu h_{i}}\right)=\prod_{i=1}^{k}\left(\frac{1}{1-x_{i}^{\nu}}\right) .
$$

We study now the case when $\nu \equiv a \bmod d$. The generic contribution of the parts $\nu$ may be written in the following way:

$$
x_{1}^{\nu h_{1}} \cdots x_{k}^{\nu h_{k}}\left(z^{h}\left(x_{1} \cdots x_{k}\right)^{h \nu}\right)
$$

where at least one exponent $h_{i}=0$. Such terms correspond to the partitions of some $n_{1}, \ldots, n_{k}$ such that the summand $\nu$ occurs exactly $h$ times in the intersection of $\overline{\Pi_{1}\left(n_{1}\right)} \cap \cdots \cap \overline{\Pi_{k}\left(n_{k}\right)}$. We deduce that the contribution of such $\nu$ to the function $G$ is

$$
\begin{aligned}
g_{2}(\nu) & :=\left(\sum_{\substack{I \subset\{1, \ldots, k\} \\
|I| \neq k}} \prod_{i \in I}\left(\sum_{h=1}^{\infty} x_{i}^{h \nu}\right)\right)\left(1+\sum_{h=1}^{\infty} z^{h} \prod_{i=1}^{k} x_{i}^{\nu h}\right) \\
& =\left(\frac{1-\prod_{i=1}^{k} x_{i}^{\nu}}{\prod_{i=1}^{k}\left(1-x_{i}^{\nu}\right)}\right)\left(\frac{1}{1-z \prod_{i=1}^{k} x_{i}^{\nu}}\right) .
\end{aligned}
$$

By (6) and (8) we deduce that

$$
G(\mathbf{x}, z)=\prod_{\substack{\nu \geq 1 \\ \nu \neq a}} g_{1}(\nu) \prod_{\substack{\nu \geq 1 \\ \nu \equiv a}} g_{2}(\nu),
$$


this ends the proof of Lemma 1.

Now we compute the coefficient of $z^{m}$ in $G(\mathbf{x}, z)$. If we write $G(\mathbf{x}, z)=$ $\sum_{m=0}^{\infty} g(\mathbf{x}, m) z^{m}$ then we have:

$$
g(\mathbf{x}, m)=\sum_{n_{1}=0}^{\infty} \ldots \sum_{n_{k}=0}^{\infty} \alpha\left(n_{1}, \ldots, n_{k} ; m ; a, d\right) x_{1}^{n_{1}} \cdots x_{k}^{n_{k}} .
$$

These coefficient $g(\mathbf{x}, m)$ have the following form:

Lemma 2. For $\left|x_{1}\right|<1, \ldots,\left|x_{k}\right|<1,\left|x_{1}^{a} \cdots x_{k}^{a} z\right|<1, m \in \mathbb{N}$, we have

$g(\mathbf{x}, m)=\left\{\prod_{\nu=1}^{\infty} \prod_{i=1}^{k} \frac{1}{1-x_{i}^{\nu}}\right\}\left\{\prod_{\mu=0}^{\infty}\left(1-\left(x_{1} \cdots x_{k}\right)^{a+d \mu}\right)\right\} \frac{\left(x_{1} \cdots x_{k}\right)^{a m}}{\prod_{j=1}^{m}\left(1-\left(x_{1} \cdots x_{k}\right)^{j d}\right)}$.

Proof. We will use the following formula (for example, see [10] Theorem 349 p. 280)

Lemma 3 (Euler ). For $|\lambda|<1,|\xi \lambda|<1$ we have

$$
\prod_{\mu=1}^{\infty} \frac{1}{1-\xi \lambda^{\mu}}=\sum_{\mu=0}^{\infty} c_{\mu}(\lambda) \xi^{\mu}
$$

with

$$
c_{\mu}(\lambda)=\frac{\lambda^{\mu}}{(1-\lambda)\left(1-\lambda^{2}\right) \cdots\left(1-\lambda^{\mu}\right)}
$$

The first product in the expression of $G(\mathbf{x}, z)$ given in Lemma 1 doesn't depend on $z$, thus we only need to study the second product with the $\nu \equiv a$ $\bmod d$. Writing $\nu=a+\mu d$ we have

$$
\prod_{\nu \equiv a, \bmod } \frac{1-\left(x_{1} \cdots x_{k}\right)^{\nu}}{1-\left(x_{1} \cdots x_{k}\right)^{\nu} z}=\prod_{\mu=0}^{\infty} \frac{1-\left(x_{1} \cdots x_{k}\right)^{a}\left(\left(x_{1} \cdots x_{k}\right)^{d}\right)^{\mu}}{1-\left(x_{1} \cdots x_{k}\right)^{a} z\left(\left(x_{1} \cdots x_{k}\right)^{d}\right)^{\mu}} .
$$

Then we apply Euler's formula (Lemma 3) to expand the denominator

$$
\begin{aligned}
& \prod_{\substack{\nu \geq 1 \\
\nu \equiv a}} \frac{1-\left(x_{1} \cdots x_{k}\right)^{\nu}}{1-\left(x_{1} \cdots x_{k}\right)^{\nu} z}=\left(\prod_{\mu=0}^{\infty} 1-\left(x_{1} \cdots x_{k}\right)^{a+d \mu}\right) \\
& \times \sum_{m=0}^{\infty} \frac{\left(\left(x_{1} \cdots x_{k}\right)^{a} z\right)^{m}}{\prod_{j=1}^{m}\left(1-\left(x_{1} \cdots x_{k}\right)^{j d}\right)} .
\end{aligned}
$$

This ends the proof of Lemma 2 .

\section{Proof of Theorem 1}

Let $A\left(n_{1}, \ldots, n_{k} ; M ; a, d\right)=\sum_{m=0}^{M} \alpha\left(n_{1}, \ldots, n_{k} ; m, a, d\right)$ the number of $k$-tuples of partitions with few common summands $\equiv a \bmod d$. To prove Theorem 1 it is sufficient to show that

$$
A\left(n_{1}, \ldots, n_{k} ; M ; a, d\right)=o\left(p\left(n_{1}\right) \cdots p\left(n_{k}\right)\right)
$$


when $M \leq(1-\delta)(\sqrt{6} /(2 \pi k d)) \sqrt{n} \log n$.

By Lemma 2, we have:

$$
\begin{aligned}
\sum_{n_{1}=0}^{\infty} \cdots \sum_{n_{k}=0}^{\infty} & A\left(n_{1}, \ldots, n_{k} ; M ; a, d\right) \prod_{i=1}^{k} x_{i}^{n_{i}}=\left(\prod_{\nu=1}^{\infty} \prod_{i=1}^{k} \frac{1}{1-x_{i}^{\nu}}\right) \\
& \times\left(\prod_{\mu=0}^{\infty}\left(1-\left(\prod_{i=1}^{k} x_{i}\right)^{a+d \mu}\right)\right) \sum_{m=0}^{M} \frac{\left(x_{1} \cdots x_{k}\right)^{a m}}{\prod_{j=1}^{m}\left(1-\left(x_{1} \cdots x_{k}\right)^{j d}\right)} .
\end{aligned}
$$

For $0<x_{1}, \ldots, x_{k}<1$, we get:

$$
\begin{aligned}
A\left(n_{1}, \ldots, n_{k} ; M ; a, d\right) \prod_{i=1}^{k} x_{i}^{n_{i}} & \leq\left(\prod_{\nu=1}^{\infty} \prod_{i=1}^{k} \frac{1}{1-x_{i}^{\nu}}\right)\left(\prod_{\mu=0}^{\infty}\left(1-\left(x_{1} \cdots x_{k}\right)^{a+d \mu}\right)\right) \\
& \times \sum_{m=0}^{M} \frac{\left(x_{1} \cdots x_{k}\right)^{a m}}{\prod_{j=1}^{m}\left(1-\left(x_{1} \cdots x_{k}\right)^{j d}\right)} .
\end{aligned}
$$

Since $\left.x_{i} \in\right] 0,1[$ the sum for $0 \leq m \leq M$ is less than

$$
\sum_{m=0}^{M} \frac{\left(x_{1} \cdots x_{k}\right)^{a m}}{\prod_{j=1}^{M}\left(1-\left(x_{1} \cdots x_{k}\right)^{j d}\right)} \leq \frac{1}{\left(1-\left(x_{1} \cdots x_{k}\right)^{a}\right) \prod_{j=1}^{M}\left(1-\left(x_{1} \cdots x_{k}\right)^{j d}\right)} .
$$

The factor $\left(1-\left(x_{1} \cdots x_{k}\right)^{a}\right)$ in the previous fraction appears also in the product in $\mu$ in (10). Thus we can remove the factor $\mu=0$ in this product. Next we use the fact that $1-\left(x_{1} \cdots x_{k}\right)^{a+d \mu} \leq 1-\left(x_{1} \cdots x_{k}\right)^{d(\mu+1)}$ for all $\mu \geq 1$. With this trick we find some simplifications with the product in the variable $j$ in (11). After all these manipulations we get:

$$
\begin{aligned}
A\left(n_{1}, \ldots, n_{k} ; M ; a, d\right) x_{1}^{n_{1}} \cdots x_{k}^{n_{k}} & \leq \frac{1}{1-\left(x_{1} \cdots x_{k}\right)^{d}}\left(\prod_{\nu=1}^{\infty} \prod_{j=1}^{k} \frac{1}{1-x_{j}^{\nu}}\right) \\
& \times\left(\prod_{\mu=M+1}^{\infty}\left(1-\left(x_{1} \cdots x_{k}\right)^{d \mu}\right)\right) .
\end{aligned}
$$

Let $f(z)=\prod_{\nu=1}^{\infty}\left(1-\mathrm{e}^{-\nu z}\right)^{-1}$ for $\Re e z>0$. Choosing $x_{j}=\mathrm{e}^{-t_{j}}, t_{j}>0$, $j=1, \ldots, k$ we have:

$$
\begin{aligned}
A\left(n_{1}, \ldots, n_{k} ; M ; a, d\right) \exp \left(-\sum_{i=1}^{k} n_{i} t_{i}\right) & \leq \frac{\prod_{i=1}^{k} f\left(t_{i}\right)}{1-\exp \left(-d\left(\sum_{i=1}^{k} t_{i}\right)\right)} \\
& \times \prod_{\mu=M+1}^{\infty}\left(1-\exp \left(-d \mu\left(\sum_{i=1}^{k} t_{i}\right)\right)\right) .
\end{aligned}
$$

Next we use the following inequalities :

$$
\frac{1}{1-\exp \left(-d\left(\sum_{i=1}^{k} t_{i}\right)\right.}=\frac{\exp \left(d \sum_{i=1}^{k} t_{i}\right)}{\exp \left(d \sum_{i=1}^{k} t_{i}\right)-1} \leq \frac{\exp \left(d \sum_{i=1}^{k} t_{i}\right)}{\sum_{i=1}^{k} d t_{i}}
$$


and

$$
\begin{aligned}
\prod_{\mu=M+1}^{\infty}\left(1-\exp \left(-d \mu \sum_{i=1}^{k} t_{i}\right)\right) & =\exp \left(-\sum_{\mu=M+1}^{\infty} \log \frac{1}{1-\exp \left(-d \mu \sum_{i=1}^{k} t_{i}\right)}\right) \\
& \leq \exp \left(-\sum_{\mu=M+1}^{\infty} \exp \left(-d \mu \sum_{i=1}^{k} t_{i}\right)\right) \\
& =\exp \left(-\frac{\exp \left(-d(M+1) \sum_{i=1}^{k} t_{i}\right)}{1-\exp \left(-d \sum_{i=1}^{k} t_{i}\right)}\right) .
\end{aligned}
$$

We obtain

$$
\begin{aligned}
A\left(n_{1}, \ldots, n_{k} ; M ; a, d\right) \exp \left(-\sum_{i=1}^{k} n_{i} t_{i}\right) & \leq \frac{\exp \left(d \sum_{i=1}^{k} t_{i}\right)}{d \sum_{i=1}^{k} t_{i}} \prod_{i=1}^{k} f\left(t_{i}\right) \\
& \times \exp \left(-\frac{\exp \left(-d(M+1) \sum_{i=1}^{k} t_{i}\right)}{1-\exp \left(-d \sum_{i=1}^{k} t_{i}\right)}\right) .
\end{aligned}
$$

Since for $t>0, t \rightarrow+0, f(t)=(1+o(1)) \sqrt{\frac{t}{2 \pi}} \exp \left(\frac{\pi^{2}}{6 t}\right)$, we obtain for $\max _{1 \leq j \leq k} t_{j} \rightarrow+0$

$$
\begin{aligned}
A\left(n_{1}, \ldots, n_{k} ; M ; a, d\right) & \ll \frac{\left(\prod_{i=1}^{k} t_{i}\right)^{1 / 2}}{d \sum_{i=1}^{k} t_{i}} \exp \left(\sum_{i=1}^{k}\left(n_{i} t_{i}+\frac{\pi^{2}}{6 t_{i}}\right)\right. \\
& \left.-(1+o(1)) \frac{\exp \left(-d M \sum_{i=1}^{k} t_{i}\right)}{d \sum_{i=1}^{k} t_{i}}\right) .
\end{aligned}
$$

Finally, let $t_{j}=\pi / \sqrt{6 n_{j}}, j=1, \ldots, k$. Then for $n \rightarrow \infty$,

$$
n(1+o(1)) \leq n_{1} \leq \ldots \leq n_{k} \leq n(1+o(1))
$$

we have:

$$
\begin{aligned}
& A\left(n_{1}, \ldots, n_{k} ; M ; a, d\right) \\
& \ll n^{\frac{3 k}{4}+\frac{1}{2}} \exp \left(-(1+o(1)) \frac{\exp (-(1+o(1)) d M k \pi / \sqrt{6 n})}{d k \pi / \sqrt{6 n}}\right) \prod_{i=1}^{k} p\left(n_{i}\right) \\
& \ll \exp \left(\frac{3 k+2}{4} \log n-(1+o(1)) \frac{\exp (-(1+o(1)) d M k \pi / \sqrt{6 n})}{d k \pi / \sqrt{6 n}}\right) \prod_{i=1}^{k} p\left(n_{i}\right) .
\end{aligned}
$$

This upper bound is $o\left(p\left(n_{1}\right) \cdots p\left(n_{k}\right)\right)$ if $M=[(1-\delta)(\sqrt{6 n} /(2 \pi k d)) \log n]$ with a fixed, small positive $\delta$. This ends the proof of Theorem 1 .

\section{The saddle point method}

We focus now on the case $k=2, n_{1}=n_{2}=n$. By Lemma 2 and the Cauchy formula we have for any $0<\delta, \varrho<1$ :

$$
\begin{aligned}
\alpha(n, n ; M ; a, d) & =\frac{1}{(2 i \pi)^{2}} \int_{\left|x_{1}\right|=\delta} \int_{\left|x_{2}\right|=\varrho}\left(\prod_{\nu=1}^{\infty} \frac{1}{\left(1-x_{1}^{\nu}\right)\left(1-x_{2}^{\nu}\right)}\right) \\
& \times\left\{\prod_{\mu=0}^{\infty}\left(1-\left(x_{1} x_{2}\right)^{a+d \mu}\right)\right\} \frac{x_{1}^{a M-n_{1}-1} x_{2}^{a M-n_{2}-1}}{\prod_{j=1}^{M}\left(1-\left(x_{1} x_{2}\right)^{j d}\right)} \mathrm{d} x_{1} \mathrm{~d} x_{2} .
\end{aligned}
$$


As it is usual for partition problems we write $x_{1}=\mathrm{e}^{-z_{1}}, x_{2}=\mathrm{e}^{-z_{2}}$, with $z_{1}=x+i y, z_{2}=x+i t, x=\frac{\pi}{\sqrt{6 n}}$. Then we have

$$
\begin{aligned}
\alpha(n, n ; M ; a, d) & =\frac{1}{4 \pi^{2}} \int_{-\pi}^{\pi} \int_{-\pi}^{\pi} f(x+i y) f(x+i t) \\
& \times \frac{\prod_{\mu=0}^{\infty}(1-\exp (-(a+d \mu)(2 x+i(y+t))))}{\prod_{j=1}^{M}(1-\exp (-d j(2 x+i(y+t))))} \\
& \times \exp ((n-a M)(2 x+i(y+t))) \mathrm{d} y \mathrm{~d} t .
\end{aligned}
$$

Next we split the integral in two ranges. The main contribution will come from the square $\max (|y|,|t|) \leq 2 \pi x$.

\section{Some trivial upper bounds for all the range $-\pi \leq y, t \leq \pi$}

A standard way to handle the generating function $f$ is to write this product as the sum of logarithms in the exponent, next to expand in analytic series the logarithms and finally to exchange the order of summations and compute some geometric series:

$$
f(x+i y) f(x+i t)=\exp \left(\sum_{m=1}^{\infty} \frac{1}{m}\left(\frac{1}{\exp (m(x+i y))-1}+\frac{1}{\exp (m(x+i t)-1}\right)\right) .
$$

Next for every $y \in[-\pi, \pi]$, we have $|\exp (m(x+i y))-1| \geq \mathrm{e}^{m x}-1 \geq$ $m x$. Thus for every $-\pi \leq y, t \leq \pi$ we have:

$$
|f(x+i y) f(x+i t)| \leq \exp \left(2 \sum_{m=1}^{\infty} \frac{1}{m^{2} x}\right)=\exp \left(\frac{\pi^{2}}{3 x}\right)=\exp \left(\frac{2 \pi \sqrt{n}}{\sqrt{6}}\right) .
$$

Now we examine the ratio of products in (12). Let $R(y, t)$ denote this ratio:

$$
R(y, t)=\frac{\prod_{\mu=0}^{\infty}(1-\exp (-(a+d \mu)(2 x+i(y+t))))}{\prod_{j=1}^{M}(1-\exp (-d j(2 x+i(y+t))))} .
$$

By some trivial upper bounds we have:

$$
|R(y, t)| \leq \frac{\left(1+\mathrm{e}^{-2 a x}\right) \prod_{\mu=1}^{\infty}(1+\exp (-2 d \mu x))}{\prod_{j=1}^{M}(1-\exp (-2 d j x))} .
$$

In the denominator we may take $M=\infty$ if we want only an upper bound. The resulting quantity may then be written as the quotient of two values of $f$ :

$$
|R(y, t)| \leq 2 \frac{\prod_{\mu=1}^{\infty}\left\{(1-\exp (-4 d \mu x))(1-\exp (-2 d \mu x))^{-1}\right\}}{\prod_{j=1}^{\infty}(1-\exp (-2 d j x))}=\frac{2 f^{2}(2 d x)}{f(4 d x)} .
$$

Next we use the well-known formula (see for example [8])

$$
f(w)=\exp \left(\frac{\pi^{2}}{6 w}+\frac{1}{2} \log \frac{w}{2 \pi}+O(|w|)\right)
$$


for $w \rightarrow 0$ in $|\arg w|<\pi / 2$ and $\mathbb{R e} w>0$. We obtain:

$$
|R(y, t)| \leq(\sqrt{2}+o(1)) \sqrt{\frac{d x}{\pi}} \exp \left(\frac{\pi^{2}}{8 d x}\right) .
$$

It remains to handle one factor in (12):

$$
|\exp ((n-a M)(2 x+i(y+t)))|<\exp (2 n x)=\exp \left(\frac{2 \pi \sqrt{n}}{\sqrt{6}}\right) .
$$

These upper bounds yield to:

$$
\alpha(n, n ; M ; a, d) \ll n^{2} p^{2}(n) \exp \left(\frac{\pi^{2}}{8 d x}\right)=o\left(p^{2}(n)\right) \exp \left(\frac{5}{4 d x}\right) .
$$

When $a=d$ we can easily obtain a better result for the fraction $R(y, t)$ because there are some cancellations between the product in numerator and the product in denominator:

$$
\begin{aligned}
|R(y, t)| & =\frac{\prod_{\mu=0}^{\infty}|1-\exp (-d(\mu+1)(2 x+i(y+t)))|}{\prod_{j=1}^{M}|1-\exp (-d j(2 x+i(y+t)))|} \\
& =\prod_{j=M+1}^{\infty}|1-\exp (-d j(2 x+i(y+t)))| .
\end{aligned}
$$

With trivial upper bounds we obtain

$$
|R(y, t)| \leq \prod_{j=1}^{\infty}(1+\exp (-2 d j x))=\frac{f(2 d x)}{f(4 d x)} \sim \frac{1}{\sqrt{2}} \exp \left(\frac{\pi^{2}}{24 d x}\right) .
$$

Thus we obtain

$$
\alpha(n, n ; M ; d, d) \leq o\left(p^{2}(n)\right) \exp \left(\frac{5}{12 d x}\right) .
$$

\section{The range $2 \pi x \leq|y| \leq \pi$}

In this section we prove the following lemma

Lemma 4. With the notations $f$ and $R$ of the previous sections, we have:

$$
\begin{aligned}
\alpha(n, n ; M ; a, d) & =\frac{1}{4 \pi^{2}} \int_{-2 \pi x}^{2 \pi x} \int_{-2 \pi x}^{2 \pi x} f(x+i y) f(x+i t) R(y, t) \\
& \times \exp ((n-a M)(2 x+i(y+t))) \mathrm{d} y \mathrm{~d} t \\
& +o\left(p(n)^{2} \exp \left(-\frac{\sqrt{6 n}}{8 \pi}\right)\right) .
\end{aligned}
$$

Let us write $c_{0}=\sqrt{6} /(8 \pi)$ for the coefficient in the exponent in the above error term. 
Proof. We start with (13) and isolate the term with $m=1$ :

$$
|f(x+i y) f(x+i t)| \leq \exp \left(\Re e \frac{1}{\exp (x+i y)-1}+\frac{1}{\mathrm{e}^{x}-1}+\sum_{m=2}^{\infty} \frac{2}{m\left(\mathrm{e}^{m x}-1\right)}\right) .
$$

Then we use the upper bound proved in [4] p. 78 :

$$
\begin{aligned}
|f(x+i y) f(x+i t)| & \leq \exp \left(\frac{\pi}{2|y|}+\frac{1}{x}+\sum_{m=2}^{\infty} \frac{2}{x m^{2}}\right) \\
& \leq \exp \left(\frac{\pi}{2|y|}-\frac{1}{x}+\frac{\pi^{2}}{3 x}\right) \\
& \leq \exp \left(\frac{\pi^{2}}{3 x}-\frac{3}{4 x}\right)
\end{aligned}
$$

when $2 \pi x \leq|y| \leq \pi$. Thus the contribution of $\int_{2 \pi x \leq|y| \leq p i} \int_{|t| \leq \pi}$ is

$$
o\left(p^{2}(n)\right) \exp \left(\frac{5}{4 d x}-\frac{3}{4 x}\right) .
$$

If $\mathrm{d} \geq 2$ we see easily that it is $o\left(p^{2}(n)\right) \mathrm{e}^{-c_{0} \sqrt{n}}$ with the $c_{0}$ defined above. If $d=1$, then $a=d=1$ : we combine (16) with (15) and this gives a contribution bounded by

$$
o\left(p^{2}(n)\right) \exp \left(\frac{5}{12 x}-\frac{9}{12 x}\right)=o\left(p^{2}(n)\right) \mathrm{e}^{-c_{0} \sqrt{n}} .
$$

The same result can be obtained for $2 \pi x \leq|t| \leq \pi,|y| \leq \pi$ and this ends the proof of Lemma 4.

\section{$7 \quad$ The special case $a=d$.}

By Lemma 4 we have

$$
\alpha(n, n ; M ; a, d)=I+o\left(p^{2}(n) \mathrm{e}^{-c_{0} \sqrt{n}}\right),
$$

where $I$ is the contribution from $\max (|y|,|t|) \leq 2 \pi x$. In the case $a=d$ the integral $I$ has an easier presentation:

$$
\begin{aligned}
I & =\frac{1}{4 \pi^{2}} \int_{-2 \pi x}^{2 \pi x} \int_{-2 \pi x}^{2 \pi x} f(x+i y) f(x+i t) \prod_{j=M+1}^{\infty}(1-\exp (-d j(2 x+i(y+t)))) \\
& \times \exp ((n-d M)(2 x+i(y+t))) \mathrm{d} y \mathrm{~d} t .
\end{aligned}
$$

Suppose that $\varepsilon>0$ and

$$
\left(\frac{3}{4}+\varepsilon\right) \frac{1}{2 d} \frac{\sqrt{6 n}}{2 \pi} \log n \leq M \leq \frac{n^{\frac{5}{8}}}{d} .
$$

For $j>M,|\exp (-d j(2 x+i(y+t)))|=\exp (-2 d j x)<\exp (-2 d M x) \leq$ $n^{-\frac{3}{8}-\frac{\varepsilon}{2}}$. In [4] we have already met a similar situation. There we worked with the functions $g_{M}(w)=f(w) \prod_{j \geq M+1}(1-\exp (-j w))$. 
Following the proof of formula (4.2) in [4] we obtain:

$$
\begin{aligned}
& \prod_{j=M+1}^{\infty}(1-\exp (-d j(2 x+i(y+t))))=\exp \left(-\frac{\exp (-2 d M x)}{2 d x}\right. \\
& \left.+O\left(\exp (-2 d M x)\left(\sqrt{n} M|y+t|+1+\frac{\sqrt{n}}{d} \exp (-2 d M x)\right)\right)\right) .
\end{aligned}
$$

When $n^{-5 / 8+\varepsilon / 3} \leq|y| \leq 2 \pi x$ (or $n^{-5 / 8+\varepsilon / 3} \leq|t| \leq 2 \pi x$ ) the terms in $f(x+i y)$ give an extra factor $\exp \left(-n^{1 / 4}\right)$ (see [4] p. 77). Therefore

$$
\begin{aligned}
\alpha(n, n ; M ; d, d) & =\frac{1}{4 \pi^{2}} \iint_{\max (|y|,|t|) \leq n^{-\frac{5}{8}+\frac{\varepsilon}{3}}} f(x+i y) f(x+i t) \\
& \times \prod_{j=M+1}^{\infty}(1-\exp (-d j(2 x+i(y+t)))) \\
& \times \exp ((n-d M)(2 x+i(y+t))) \mathrm{d} y \mathrm{~d} t+o\left(p^{2}(n)\right) \mathrm{e}^{-c_{1} n^{1 / 4}} .
\end{aligned}
$$

The range $n^{-3 / 4+\varepsilon / 3} \leq|y| \leq n^{-5 / 8+\varepsilon / 3}$ (or $n^{-3 / 4+\varepsilon / 3} \leq|t| \leq n^{-5 / 8+\varepsilon / 3}$ ) can be settled as in [4] pp. $76-77$ :

$$
\begin{aligned}
\alpha(n, n ; M ; d, d) & =\frac{1+o(1)}{4 \pi^{2}} \iint_{|y|,|t| \leq n}{ }^{-\frac{3}{4}+\frac{\varepsilon}{3}} f(x+i y) f(x+i t) \\
& \times \exp \left(-\frac{\exp (-2 d M x)}{2 d x}\right) \\
& \times \exp \left((n-d M)(2 x+i(y+t)) \mathrm{d} y \mathrm{~d} t+O\left(p^{2}(n) \mathrm{e}^{-c_{1} n^{1 / 4}}\right) .\right.
\end{aligned}
$$

After some simplifications we apply (14)

$$
\begin{aligned}
\alpha(n, n ; M ; d, d) & =\frac{1+o(1)}{4 \pi^{2}} \exp \left(-\frac{\exp -2 d M x}{2 d x}-2 d M x\right) \\
& \times\left(\int_{|y| \leq n^{-\frac{3}{4}+\frac{\varepsilon}{3}}} f(x+i y) \exp (n(x+i y)) \mathrm{d} y\right)^{2} \\
& +O\left(p^{2}(n) \mathrm{e}^{-c_{1} n^{1 / 4}}\right) \\
& =(1+o(1)) p^{2}(n) \exp \left(-\frac{\exp (-2 d M x)}{2 d x}-2 d M x\right)
\end{aligned}
$$

since

$$
\exp \left(\frac{\exp (-2 d M x)}{2 d x}+2 d M x\right) \leq \exp \left(c_{2} n^{1 / 8}\right)
$$

\section{The general case $1 \leq a \leq d$}

As in the case $a=d$ we apply Lemma 4 :

$$
\alpha(n, n ; M, a, d)=I+o\left(p^{2}(n) \mathrm{e}^{-c_{0} \sqrt{n}}\right),
$$

but in this general case, the integral $I$ has a more complicated form:

$$
\begin{aligned}
I & =\frac{1}{4 \pi^{2}} \int_{-2 \pi x}^{2 \pi x} \int_{-2 \pi x}^{2 \pi x} f(x+i y) f(x+i t) D(2 x+i(y+t)) \\
& \times \prod_{j=M+1}^{\infty}(1-\exp (-d j(2 x+i(y+t)))) \exp ((n-a M)(2 x+i(y+t)) \mathrm{d} y \mathrm{~d} t .
\end{aligned}
$$


We recognize the formula in the case $a=d$ but with a disruptive quotient $D(2 x+i(y+t))$ in addition

$$
D(w)=\frac{\prod_{\mu=0}^{\infty}(1-\exp (-(a+d \mu) w))}{\prod_{j=1}^{\infty}(1-\exp (-d j w))} .
$$

We will now obtain some convenient upper bounds for $D(2 x+i(y+t))$ when $\max (|y|,|t|)>n^{-3 / 4+\varepsilon / 3}$ and asymptotic estimates for the range $\max (|y|,|t|) \leq n^{-3 / 4+\varepsilon / 3}$. Then the proof of Theorem 2 will be a consequence of these estimations and the work done in the previous section for the case $a=d$.

Lemma 5. For $|y|,|t| \leq 2 \pi x$, we have

$$
|D(2 x+i(y+t))|=\exp (O(\log n)) .
$$

Proof. In (18) we change the index of the denominator in order to start with $j=0$ instead of 1 . Next we do some suitable manipulations which transform the quotients $(1-\ldots) /(1-\ldots)$ in terms like $1-\ldots$ :

$$
D(2 x+i(y+t))=\prod_{j=0}^{\infty}\left(1-\frac{\exp ((d-a)(2 x+i(y+t)))-1}{\exp ((d+d j)(2 x+i(y+t)))-1}\right) .
$$

Now the numerator above is independent of $j$ :

$$
|D(2 x+i(y+t))| \leq \prod_{\mu=1}^{\infty}\left(1+\frac{|\exp ((d-a)(2 x+i(y+t)))-1|}{\exp (2 d \mu x)-1}\right) .
$$

When $d$ is fixed, $|\exp ((d-a)(2 x+i(y+t)))-1|=O\left(n^{-1 / 2}\right)$. By the classical formula $1+u \leq \mathrm{e}^{u}(u \in \mathbb{R})$ we obtain

$$
|D(2 x+i(y+t))| \leq \exp \left(\sum_{\mu=1}^{\infty} \frac{O\left(n^{-1 / 2}\right)}{\exp (2 \mu d x)-1}\right) .
$$

We split the summation over $\mu$ in two parts. When $\mu$ is small that is $\mu \leq 1 /(2 d x)$, we use the fact that $\exp (2 \mu d x)-1 \geq 2 d \mu x$ otherwise we use the fact that $(1-\exp (-2 d \mu x))^{-1}=O(1)$ :

$$
\begin{aligned}
\sum_{\mu=1}^{\infty} \frac{1}{\exp (2 \mu d x)-1} & \leq \sum_{\mu \leq \frac{1}{2 d x}} \frac{1}{2 d x \mu}+\sum_{\mu>\frac{1}{2 d x}} \frac{\exp (-2 d \mu x)}{1-\exp (-2 d \mu x)} \\
& \ll \frac{1}{2 d x} \log \frac{1}{2 d x}+\sum_{\mu=1}^{\infty} \mathrm{e}^{-2 d \mu x} \ll \frac{1}{2 d x} \log \frac{1}{2 d x}
\end{aligned}
$$

It remains to insert (21) in (20) to finish the proof of Lemma 5.

The upper bound of Lemma 5 is small enough to deduce with the computations of the previous section that the contribution of the range $n^{-5 / 8+\varepsilon / 3} \leq \max (|y|,|t|) \leq 2 \pi x$ is $O\left(p^{2}(n)\right) \mathrm{e}^{-c_{1}^{\prime} n^{1 / 4}}$ for some $c_{1}^{\prime}>0$.

Now we study the case $\max (|y|,|t|) \leq n^{-5 / 8+\varepsilon / 3}$. 
Lemma 6. For $\max (|y|,|t|) \leq n^{-5 / 8+\varepsilon / 3}$ the modifying factor satisfies

$$
D(2 x+i(y+t))=\left(1+O\left(n^{-\frac{1}{8}+\varepsilon}\right)\right) \prod_{\mu=1}^{\infty}\left(1-\frac{\exp ((d-a) 2 x)-1}{\exp (2 \mu d x)-1}\right) .
$$

Proof. The challenge of the proof is to obtain an approximation of $D(2 x+$ $i(y+t))$ by an expression independent of $y$ and $t$. We recall the expression of $D$ :

$$
D(2 x+i(y+t))=\prod_{\mu=1}^{\infty}\left(1-\frac{\exp ((d-a)(2 x+i(y+t)))-1}{\exp (d \mu(2 x+i(y+t)))-1}\right) .
$$

We will proceed in two steps. In the first step we will compare $D(2 x+$ $i(y+t))$ with a similar product but with no $y$ and $t$ in the numerators. We will prove that

$$
\frac{\prod_{\mu=1}^{\infty}\left(1-\frac{\exp ((d-a)(2 x+i(y+t)))-1}{\exp (d \mu(2 x+i(y+t)))-1}\right)}{\prod_{\mu=1}^{\infty}\left(1-\frac{\exp ((d-a) 2 x))-1}{\exp (d \mu(2 x+i(y+t)))-1}\right)}=1+O\left(n^{-\frac{1}{8}+\varepsilon}\right) .
$$

The second step consists of removing $y$ and $t$ in the denominators, that is to prove:

$$
\frac{\prod_{\mu=1}^{\infty}\left(1-\frac{\exp ((d-a) 2 x)-1}{\exp (d \mu(2 x+i(y+t)))-1}\right)}{\prod_{\mu=1}^{\infty}\left(1-\frac{\exp ((d-a) 2 x)-1}{\exp (2 d x \mu)-1}\right)}=1+O\left(n^{-\frac{1}{8}+\varepsilon}\right) .
$$

Lemma 6 will be a consequence of (22) and (23).

We now prove (22). Le $T_{1}$ denote the left hand side of this formula. As in the proof of Lemma 5 , we write each term in a product of type $1-\ldots$ :

$$
T_{1}=\prod_{\mu=1}^{\infty}\left(1-\frac{\frac{\exp ((d-a)(2 x+i(y+t)))-\exp (2 x(d-a))}{\exp (d \mu(2 x+i(y+t)))-1}}{1-\frac{\exp (2 x(d-a))-1}{\exp (d \mu(2 x+i(y+t)))-1}}\right)
$$

The denominators $\exp (d \mu(2 x+i(y+t)))-1$ are cancelling, next we factorize with $\exp ((d-a) 2 x)$, this gives:

$$
T_{1}=\prod_{\mu=1}^{\infty}\left(1-\frac{\exp ((d-a) i(y+t))-1}{\exp ((d \mu-d+a) 2 x+i d \mu(y+t))-1}\right) .
$$

Next we proceed as in the previous sections, we write the product as the sum of the logarithms in the exponent and use some standard inequalities:

$$
T_{1}=\exp \left(-\sum_{\mu=1}^{\infty} \log \frac{1}{1-\frac{\exp ((d-a) i(y+t))-1}{\exp ((d \mu-d+a) 2 x+i d \mu(y+t))-1}}\right)
$$


This gives the upper bound

$$
\begin{aligned}
T_{1} & =\exp \left(O\left(\sum_{\mu=1}^{\infty}\left|\frac{\exp ((d-a) i(y+t))-1}{\exp ((d \mu-d+a) 2 x+i d \mu(y+t))-1}\right|\right)\right) \\
& =\exp \left(O(1) \sum_{\mu=1}^{\infty} \frac{(d-a) \mid y+t]}{\exp ((d \mu-d+a) 2 x)-1}\right) \\
& =\exp \left(O\left(n^{-\frac{1}{8}+\varepsilon}\right)\right)=1+O\left(n^{-\frac{1}{8}+\varepsilon}\right) .
\end{aligned}
$$

This ends the proof of (22). We now prove (23). Let $T_{2}$ denote the left hand side of this formula. For $1 \leq a<d$ we start in the same way as for $T_{1}$ :

$$
T_{2}=\prod_{\mu=1}^{\infty}\left(1-\frac{\frac{\exp (2 x(d-a))-1}{\exp (d \mu(2 x+i(y+t)))-1}-\frac{\exp (2 x(d-a))-1}{\exp (2 d \mu x)-1}}{1-\frac{\exp (2 x(d-a))-1}{\exp (2 d \mu x)-1}}\right) .
$$

We factor out $\exp (2 x(d-a))-1$ and/or simplify by it:

$$
T_{2}=\prod_{\mu=1}^{\infty}\left(1-\frac{\frac{1}{\exp (d \mu(2 x+i(y+t)))-1}-\frac{1}{\exp (2 \mu d x)-1}}{\frac{1}{\exp ((d-a) 2 x)-1}-\frac{1}{\exp (2 \mu d x)-1}}\right) .
$$

We put in the same denominator the two differences of fractions and next we factor out $\exp (2 d \mu x)$. After these two manipulations we obtain:

$$
T_{2}=\prod_{\mu=1}^{\infty}\left(1-\frac{\frac{1-\exp (d \mu i(y+t))}{\exp (d \mu(2 x+i(y+t))-1}}{\frac{1-\exp (-(d \mu-d+a) 2 x)}{\exp ((d-a) 2 x)-1}}\right)
$$

We are now ready to do some standard upper bounds using the same type of ideas as in $T_{1}$ :

$$
T_{2}=\exp \left(O\left(\sum_{\mu=1}^{\infty}\left|\frac{\frac{1-\exp (d \mu i(y+t))}{\exp (d \mu(2 x+i(y+t))-1}}{\frac{1-\exp (-(d \mu-d+a) 2 x)}{\exp ((d-a) 2 x)-1}}\right|\right)\right)
$$

Thus we obtain

$$
T_{2}=\exp \left(O(1) \sum_{\mu=1}^{\infty} \frac{d \mu|y+t|(\exp (2 x(d-a))-1)}{(\exp (2 d \mu x)-1)(1-\exp (-(d \mu-d+a) 2 x))}\right) .
$$

As in the end of the proof of Lemma 5 we split the sum on $\mu$ in two sums and handle these sums as before:

$$
\begin{aligned}
T_{2} & =\exp \left(O(1) \sum_{\mu \leq 1 /(2 d x)} \frac{d \mu|y+t| 2 x(d-a)}{2 \mu d x(d \mu-d+a) 2 x}+O(1) \sum_{\mu>1 /(2 d x)} \frac{\mu|y+t| d^{2} x}{\exp (2 \mu d x)}\right) \\
& =\exp \left(O(1)|y+t| \sqrt{n} \log n+O(1) d^{2}|y+t| x \sum_{\mu=1}^{\infty} \mu(\exp (-2 d x))^{\mu}\right) .
\end{aligned}
$$


The last sum in $\mu$ is $O\left(1 / x^{2}\right)$. Thus we obtain

$$
T_{2}=\exp \left(O\left(n^{-\frac{1}{8}+\varepsilon}\right)\right)=1+O\left(n^{-\frac{1}{8}+\varepsilon}\right) .
$$

This ends the proof of (23).

\section{The modifying factor}

Let $P(2 x)$ denote the product in the right hand side of the formula of Lemma 6 . We can write this product in the following way:

$$
P(2 x)=f(2 d x) \prod_{\substack{n \geq 1 \\ n \equiv a}}(1-\exp (-2 x n))=f(2 d x) Q(2 x),
$$

say. The product $Q(x)^{-1}$ is the generating series associated with partitions with parts $\equiv a \bmod d$. It appears in [11] as the first example of application of the general result of Meinardus. Following his method we find when $\sigma \in \mathbb{R}, \sigma \rightarrow 0$ :

$$
Q(\sigma)^{-1}=(1+O(\sigma)) \exp \left(\frac{\pi^{2}}{6 d \sigma}\right) \Gamma\left(\frac{a}{d}\right) \frac{(d \sigma)^{a / d-1 / 2}}{\sqrt{2 \pi}} .
$$

Thus we obtain:

$$
P(2 x)=(1+O(x)) \frac{(2 d x)^{1-a / d}}{\Gamma(a / d)}=\left(1+O\left(n^{-1 / 2}\right)\right)\left(\frac{2 \pi d}{\sqrt{6 n}}\right)^{1-a / d} \frac{1}{\Gamma(a / d)} .
$$

This ends the proof of Theorem 2 .

\section{Proofs of the corollaries}

Before proving Corollary 1 we prove the following lemma on some stability of the behaviour of $\alpha(n, n ; M, a, d)$.

Lemma 7. Let $a, d, M, \varepsilon$ satisfy all the conditions of Theorem 2. For $|t-M| \leq n^{\frac{3}{8}-2 \varepsilon}$ we have

$$
\begin{aligned}
\alpha(n, n ; M ; a, d) & =(1+o(1)) p^{2}(n)\left(\frac{2 \pi d}{\sqrt{6 n}}\right)^{1-\frac{a}{d}} \frac{1}{\Gamma\left(\frac{a}{d}\right)} \\
& \times \exp \left(-\frac{\sqrt{6 n} \exp \left(\frac{-2 \pi d t}{\sqrt{6 n}}\right)}{2 \pi d}-2 a t \frac{\pi}{\sqrt{6 n}}\right) .
\end{aligned}
$$

Proof. We consider the function

$$
H(t)=\exp \left(-\frac{\sqrt{6 n} \exp \left(\frac{-2 \pi d t}{\sqrt{6 n}}\right)}{2 \pi d}-2 a t \frac{\pi}{\sqrt{6 n}}\right) .
$$

By Theorem 2 it is sufficient to prove that $H(M)=H(t)(1+o(1))$. By a direct computation, we have:

$$
H(M)=H(t) \exp \left(-\frac{\sqrt{6 n}}{2 \pi d}\left(\exp \left(\frac{-2 \pi d M}{\sqrt{6 n}}\right)-\exp \left(\frac{-2 \pi d t}{\sqrt{6 n}}\right)\right)-\frac{2 a \pi(M-t)}{\sqrt{6 n}}\right) .
$$


Clearly we have:

$$
\exp \left(-\frac{2 a \pi(M-t)}{\sqrt{6 n}}\right)=1+O\left(|M-t| n^{-1 / 2}\right)
$$

For the difference of the two exponential we see that:

$$
\begin{aligned}
\frac{\sqrt{6 n}}{2 \pi d}\left(\exp \left(\frac{-2 \pi d M}{\sqrt{6 n}}\right)-\exp \left(\frac{-2 \pi d t}{\sqrt{6 n}}\right)\right) & =\frac{\sqrt{6 n}}{2 \pi d} \exp \left(\frac{-2 \pi d M}{\sqrt{6 n}}\right) \\
& \times\left(1-\exp \left(\frac{-2 \pi d(t-M)}{\sqrt{6 n}}\right)\right) \\
& =O\left(|t-M| \exp \left(-\frac{2 \pi d M}{\sqrt{6 n}}\right)\right) \\
& =O\left(|t-M| n^{-\frac{3}{8}+\varepsilon}\right)
\end{aligned}
$$

Proof of Corollary 1. For brevity we will write $B_{1}(n)=\left(\frac{3}{4}+\varepsilon\right) \frac{\sqrt{6 n}}{4 \pi d} \log n$ and $B_{2}(n)=\frac{n^{\frac{5}{8}}}{d}$. Let $Z(n)$ denote the number of pairs of partitions of $n$, $\left(\Pi_{1}(n), \Pi_{2}(n)\right)$ satisfying (2). By Theorem 2 and using the notation (25) we have:

$$
Z(n)=(1+o(1)) p^{2}(n)\left(\frac{2 \pi d}{\sqrt{6 n}}\right)^{1-\frac{a}{d}} \frac{1}{\Gamma\left(\frac{a}{d}\right)} \sum_{B_{1}(n) \leq m \leq B_{2}(n)} H(m) .
$$

Next following the ideas of [4] or [5], [6], we approximate the summation over $m$ by an integral. Since by Lemma 7 we have uniformly for all $m \in\left[B_{1}(n), B_{2}(n)\right]:$

$$
H(m)=(1+o(1)) \int_{m}^{m+1} H(t) \mathrm{d} t,
$$

we obtain

$$
Z(n)=(1+o(1)) p^{2}(n)\left(\frac{2 \pi d}{\sqrt{6 n}}\right)^{1-\frac{a}{d}} \frac{1}{\Gamma\left(\frac{a}{d}\right)} \int_{B_{1}(n)}^{B_{2}(n)} H(t) \mathrm{d} t .
$$

Let $I(n)$ denote the above integral on $t$. If we set $u=\exp \left(-\frac{2 \pi d t}{\sqrt{6 n}}\right)$ then we obtain

$$
I(n)=-\frac{\sqrt{6 n}}{2 \pi d} \int_{u_{1}(n)}^{u_{2}(n)} \exp \left(-\frac{u \sqrt{6 n}}{2 \pi d}\right) u^{\frac{a}{d}-1} \mathrm{~d} u,
$$

with $u_{1}(n)=\exp \left(-\frac{2 \pi d B_{1}(n)}{\sqrt{6 n}}\right), u_{2}(n)=\exp \left(-\frac{2 \pi d B_{2}(n)}{\sqrt{6 n}}\right)$.

Next we do a second change of variables, that is $v=\frac{u \sqrt{6 n}}{2 \pi d}$. We observe some simplifications in the expression of $Z(n)$ :

$Z(n)=-(1+o(1)) \frac{p^{2}(n)}{\Gamma\left(\frac{a}{d}\right)} \int_{u_{1}(n) \sqrt{6 n} /(2 \pi d)}^{u_{2}(n) \sqrt{6 n} /(2 \pi d)} \mathrm{e}^{-v} v^{a / d-1} \mathrm{~d} v=p^{2}(n)(1+o(1))$,

by [4] p. 83. This ends the proof of Corollary 1 . 
Proof of Corollary 2

By Corollary 1 , it is sufficient to evaluate $\tilde{K}(n, n ; \lambda ; a, d)$, the number of pairs of partitions $\left(\Pi_{1}(n), \Pi_{2}(n)\right)$ such that

$$
\frac{1}{d}\left(\frac{\sqrt{6 n}}{4 \pi} \log n+\lambda \sqrt{n}\right) \leq\left|\overline{\left(\Pi_{1}(n), a, d\right)} \cap \overline{\left(\Pi_{2}(n), a, d\right)}\right| \leq B_{2}(n) .
$$

Next we apply Theorem 2 for every $M \in\left[\frac{1}{d}\left(\frac{\sqrt{6 n}}{2 \pi k} \log n+\lambda \sqrt{n}\right), B_{2}(n)\right]$ and Lemma 7 to work with some truncated gamma integral as in the previous proof. We conclude with quite the same computations as in the proof of Corollary 1 . We skip the details.

\section{References}

[1] C. Dartyge and A. Sárközy, Arithmetic properties of summands of partitions, Ramanujan Journal, 8 (2004), 199-215.

[2] C. Dartyge and A. Sárközy, Arithmetic properties of summands of partitions II, Ramanujan Journal, 10 (2005), 383-394.

[3] C. Dartyge, A. Sárközy and M. Szalay, On the distribution of the summands of partitions in residue classes, Acta Math. Hungar. 109 (3) (2005), 215-237.

[4] C. Dartyge and M. Szalay, Dominant residue classes concerning the summands of partitions, Functiones et Approximatio, XXXVII.1 (2007), 65-96.

[5] C. Dartyge and M. Szalay, Local distribution of the parts of unequal partitions in arithmetic progressions I, Publ. Math. Debrecen, vol. 79/3-4 (2011), 379-393.

[6] C. Dartyge and M. Szalay, Local distribution of the parts of unequal partitions in arithmetic progressions II, Publ. Math. Debrecen, vol. 81/3-4 (2012), 453-486.

[7] P. Erdős and J. Lehner, The distribution of the number of summands in the partitions of a positive integer, Duke Math. Journal 8 (1941), 335-345;

[8] P. Erdős and M. Szalay, On the statistical theory of partitions, In: Coll. Math. Soc. János Bolyai, 34. Topics in Classical Number Theory (Budapest 1981), 397-450, North-Holland/Elsevier, 1984.

[9] G. H. Hardy and S. Ramanujan, Asymptotic formulae in combinatory analysis, Proc. London Math. Soc. 17 (1918), 75-115.

[10] G. H. Hardy and E. M. Wright, An introduction to the theory of numbers, 5th edition, Clarendon Press, Oxford, 1978.

[11] G. Meinardus, Asymptotische Aussagen über Partitionen, Math. Zeitschr. Bd. 59, (1954), 388-398.

[12] P. Turán, On some connections between combinatorics and group theory, in: Coll. Math. Soc. János Bolyai, 4. Combinatorial Theory and its Applications (Balatonfüred, 1969), 1055-1082. 
[13] P. Turán, Combinatorics, partitions, group theory, in: Colloquio Int. s. Teorie Combinatorie (Roma, 3-15 settembre 1973), Roma, Accademia Nazionale dei Lincei, 1976, Tomo II, 181-200.

[14] P. Turán, On some phenomena in the theory of partitions, Journées arithmétiques de Bordeaux, Soc. Math. de France Astérisque 24-25 (1975), 311-319. 